\title{
Systoles of hyperbolic manifolds
}

\author{
MiKHAIL V BELOLIPETSKY \\ SCOTT A THOMSON
}

\begin{abstract}
We show that for every $n \geqslant 2$ and any $\epsilon>0$ there exists a compact hyperbolic $n$-manifold with a closed geodesic of length less than $\epsilon$. When $\epsilon$ is sufficiently small these manifolds are non-arithmetic, and they are obtained by a generalised inbreeding construction which was first suggested by Agol for $n=4$. We also show that for $n \geqslant 3$ the volumes of these manifolds grow at least as $1 / \epsilon^{n-2}$ when $\epsilon \rightarrow 0$.
\end{abstract}

22E $40,53 \mathrm{C} 22$

\section{Introduction}

Let $\mathcal{H}^{n}$ denote the hyperbolic $n$-space. By a compact hyperbolic $n$-manifold we mean a quotient space $M=\Gamma \backslash \mathcal{H}^{n}$ where $\Gamma$ is a cocompact torsion-free discrete subgroup of Isom $\left(\mathcal{H}^{n}\right)$, the group of isometries of $\mathcal{H}^{n}$. The systole of a compact Riemannian manifold $M$, denoted by $\operatorname{Syst}_{1}(M)$, is the length of a closed geodesic of the shortest length in $M$. We refer to a recent monograph by M Katz [9] for more information about systoles and systolic geometry.

It is well-known that for any $\epsilon>0$ there exist 2-dimensional compact hyperbolic manifolds having a systole of length less than $\epsilon$, and examples of such manifolds of any genus $g \geqslant 2$ can be easily constructed using Teichmüller theory. A similar result for $n=3$ can be achieved using Thurston's hyperbolic Dehn surgery theorem [18, Theorem 5.8.2]. For a long time the existence of compact hyperbolic manifolds with arbitrarily short systoles in higher dimensions was an open problem. In a recent paper [1], Agol suggested a very interesting construction which solves the problem for $n=4$. His paper was a starting point for our work.

Our main result is the following:

Theorem 1.1 For every $n \geqslant 2$ and any $\epsilon>0$, there exist compact $n$-dimensional hyperbolic manifolds $M$ with $\operatorname{Syst}_{1}(M)<\epsilon$. 
The manifolds $M$ are obtained by a variant of an inbreeding construction which was first suggested by Agol for $n=4$. We simplify the critical step in the argument of [1] which makes extensive use of geometrical finiteness and related properties, and hence limits his construction to some special examples. A principal ingredient in our proof is a lemma of Margulis and Vinberg [14] which we generalise to cocompact discrete subgroups of $\operatorname{Isom}\left(\mathcal{H}^{n}\right)$. The proof of this generalised Margulis-Vinberg lemma is the main technical part of the proof of the theorem.

Systolic geometry studies relations between systole length and volume captured by isosystolic inequalities (see [9]). Our second result provides an inequality of this type for the manifolds from the proof of Theorem 1.1. To put it into context recall that for $n \geqslant 4$ (in contrast with $n=2$ and 3 ) there exist only finitely many non-isometric hyperbolic $n$-manifolds of bounded volume (see Wang [19]). Hence when $\epsilon \rightarrow 0$ for these dimensions we will necessarily have $\operatorname{Vol}(M) \rightarrow \infty$. It is natural to ask how fast the volume grows, and the following theorem gives the answer to this question for our manifolds.

Theorem 1.2 For every $n \geqslant 3$ there exists a positive constant $C_{n}$ (which depends only on $n$ ), such that the systole length and volume of the manifolds obtained in the proof of Theorem 1.1 satisfy

$$
\operatorname{Vol}(M) \geqslant \frac{C_{n}}{\operatorname{Syst}_{1}(M)^{n-2}}
$$

We can compare this inequality with the well known Gromov isosystolic inequality [9, Theorem 12.2.2], which implies

$$
\operatorname{Vol}(M) \geqslant A_{n} \operatorname{Syst}_{1}(M)^{n},
$$

where $A_{n}$ is a positive constant which depends only on $n$. When the systole of $M$ is sufficiently large Gromov's inequality gives a better bound for the volume; however, when the systole is small the inequality of Theorem 1.2 becomes stronger.

The proof of this theorem uses important recent work of Bridgeman and Kahn on orthospectra and volumes of hyperbolic $n$-manifolds [4]. In fact, we can show that it is possible to achieve that $\operatorname{Vol}(M)$ grows exactly like a polynomial in $1 / \operatorname{Syst}_{1}(M)$ (see the discussion after the proof of Theorem 1.2 and Proposition 4.2). Therefore, Theorem 1.2 captures the growth rate of the volume in our construction.

The paper is organised as follows: In Section 2, we prove Theorem 1.1 modulo the generalised Margulis-Vinberg lemma. The proof of the lemma is given in Section 3. The next section is dedicated to Theorem 1.2. We end with remarks regarding arithmeticity, commensurability and related questions in Section 5. 
Acknowledgements We thank Ian Agol and Misha Kapovich for helpful discussions and suggestions. We are also grateful to the referee for careful proofreading of the manuscript. M Belolipetsky is partially supported by EPSRC grant EP/F022662/1.

\section{Proof of Theorem 1.1}

Let $\epsilon>0$. We first obtain a hyperbolic $n$-manifold $M$ which contains a geodesic segment of length at most $\epsilon / 2$ that is orthogonal to two hypersurfaces.

Fix a totally real number field $K \subset \mathbb{R}$, and let $f$ be a non-singular $(n+1)$-ary quadratic form of signature $(n, 1)$ defined over $K$. We assume that for Galois embeddings $\sigma: K \hookrightarrow \mathbb{R}$ which are different from the original embedding, $f^{\sigma}$ is positive definite. It is well known that $\mathrm{PO}_{f}(\mathbb{R})$ is isomorphic to the group of isometries $\operatorname{Isom}\left(\mathcal{H}^{n}\right)$ of the hyperbolic $n$-space, and that $\mathrm{PO}_{f}\left(\mathcal{O}_{K}\right)\left(=\mathrm{O}_{f}\left(\mathcal{O}_{K}\right) /\{+1,-1\}\right)$ is an arithmetic lattice in $\mathrm{PO}_{f}(\mathbb{R})$, where $\mathcal{O}_{K}$ denotes the ring of integers of $K$. The lattices obtained this way (and subgroups of $\mathrm{PO}_{f}(\mathbb{R})$ which are commensurable with them) are called arithmetic subgroups of the simplest type. From now on let us assume that the degree of the field $K$ is at least 2 ; that is, $K \neq \mathbb{Q}$. Then by Godement's compactness criterion $\mathrm{PO}_{f}\left(\mathcal{O}_{K}\right)$ is cocompact. Now, by Selberg's Lemma, one can find a torsionfree subgroup $\Gamma<\operatorname{PO}_{f}\left(\mathcal{O}_{K}\right)$, of finite index. Thus $\Gamma \backslash \mathcal{H}^{n}$ is a compact hyperbolic $n$-manifold. (We refer to MacLachlan and Reid [12] for more details about arithmetic subgroups and their properties.)

Consider the vector model of hyperbolic $n$-space associated with $f$. More precisely, we can define an inner product on $\mathbb{R}^{n+1}$ by

$$
(u, v)=(u, v)_{f}=\frac{1}{2}(f(u+v)-f(u)-f(v)) .
$$

After scaling we can assume that all coefficients of $f$ are in $\mathcal{O}_{K}$, and, moreover, for any $u, v \in \mathcal{O}_{K}^{n+1}$, we have $(u, v)_{f} \in \mathcal{O}_{K}$. This does not affect the generality of the construction and will be our standing assumption. The form $f$ has signature $(n, 1)$ which implies that $(\cdot, \cdot)_{f}$ is equivalent to a standard Lorentzian inner product on $\mathbb{R}^{n+1}$, and therefore we can repeat the well-known construction of the vector model of $\mathcal{H}^{n}$ using the inner product $(\cdot, \cdot)_{f}$ (see Ratcliffe [15, Chapter 3]).

Let us choose a vector $e_{0} \in K^{n+1}$ with $f\left(e_{0}\right)>0$. The intersection $H_{0}=\left\langle e_{0}\right\rangle^{\perp_{f}} \cap \mathcal{H}^{n}$ in the ambient space $\mathbb{R}^{n+1}$ is a hyperplane in $\mathcal{H}^{n}$ and, moreover, $\Gamma_{0}=\operatorname{Isom}\left(H_{0}\right) \cap \Gamma$ is a cocompact discrete subgroup of $\operatorname{Isom}\left(H_{0}\right)$ (where we embed $\operatorname{Isom}\left(H_{0}\right) \hookrightarrow \operatorname{Isom}\left(\mathcal{H}^{n}\right)$ in the natural way). The latter holds because $\Gamma$ is defined over $K$ and $H_{0}$ is a $K-$ rational hyperplane. Now we would like to find another $K$-rational hyperplane $H_{1}$ 
which is $\epsilon / 2-$ close to $H_{0}$ but disjoint from it. This can be achieved as follows. A wellknown result of Margulis asserts that since the group $\Gamma$ is arithmetic, its commensurator is dense in $\mathrm{PO}_{f}(\mathbb{R})$ [13, Theorem 1, page 2]. Indeed, this commensurator contains $\mathrm{PO}_{f}(K)$, so we can find $\gamma \in \mathrm{PO}_{f}(K)$ with the property that $H_{1}$ (where $H_{1}=$ $\left\langle e_{1}\right\rangle^{\perp_{f}} \cap \mathcal{H}^{n}$ and $\left.e_{1}=\gamma\left(e_{0}\right)\right)$ is disjoint, but at most distance $\epsilon / 2$ away, from $H_{0}$. The hyperplane $H_{1}$ is also $K$-rational so $\Gamma_{1}=\operatorname{Isom}\left(H_{1}\right) \cap \Gamma$ is a cocompact discrete subgroup of Isom $\left(H_{1}\right)$, and both $\Gamma_{0} \backslash H_{0}$ and $\Gamma_{1} \backslash H_{1}$ are immersed into $\Gamma \backslash \mathcal{H}^{n}$ as totally geodesic hypersurfaces.

The generalised Margulis-Vinberg lemma (below) states that one can find a finite-index subgroup $\Gamma^{\prime}<\mathrm{PO}_{f}\left(\mathcal{O}_{K}\right)$ with the property that for every $h \in \Gamma^{\prime}$,

$$
\text { either } \quad h\left(H_{0}\right)=H_{0} \quad \text { or } \quad h\left(H_{0}\right) \cap\left(H_{0} \cup H_{1}\right)=\varnothing .
$$

Reversing the roles of $H_{0}$ and $H_{1}$ we can apply the lemma again to obtain a subgroup $\Gamma^{\prime \prime}$ such that for every $h \in \Gamma^{\prime \prime}$, either $h\left(H_{1}\right)=H_{1}$ or $h\left(H_{1}\right) \cap\left(H_{0} \cup H_{1}\right)=\varnothing$.

Let $\Lambda=\Gamma \cap \Gamma^{\prime} \cap \Gamma^{\prime \prime}$. Then the natural projections of $H_{0}$ and $H_{1}$ in the quotient $\Lambda \backslash \mathcal{H}^{n}$ will be properly embedded and will not intersect. Thus the manifold $L=\Lambda \backslash \mathcal{H}^{n}$ contains properly embedded totally geodesic hypersurfaces $\Lambda_{0} \backslash H_{0}$ and $\Lambda_{1} \backslash H_{1}$ (where $\left.\Lambda_{i}=\operatorname{Isom}\left(H_{i}\right) \cap \Lambda, i=0,1\right)$ which are $\epsilon / 2$-close. Let $g$ be a geodesic segment orthogonal to both of them, so as to fulfil our aim stated at the beginning of the proof.

To complete the proof, we 'cut' $L$ along the hypersurfaces $\Lambda_{0} \backslash H_{0}$ and $\Lambda_{1} \backslash H_{1}$. Retaining the connected component containing $g$ (if the cutting separates the manifold), we have a manifold $M^{\prime}$ with a totally geodesic boundary and $g$ orthogonal to this boundary. Taking the double of $M^{\prime}$ results in a closed $n$-manifold $M$, and the segment $g$ becomes a closed geodesic of $M$ of length at most $\epsilon$. This concludes the construction, and completes the proof.

\section{Generalised Margulis-Vinberg lemma}

An earlier form of the result in this section appeared in a paper by Margulis and Vinberg [14], and was also used by Kapovich, Potyagailo and Vinberg in a paper on non-coherence of lattices [8]. The generalised version here considers the case where a quadratic form is defined over a number field $K / \mathbb{Q}$ rather than being purely rational. This generalisation is necessary for dealing with cocompact lattices, and leads to a proof that uses an essentially different argument from that for $K=\mathbb{Q}$.

As before, let $K \subset \mathbb{R}$ be a totally real algebraic number field of degree $d$, and $f$ a quadratic form over $K$ of signature $(n, 1)$ and such that $f^{\sigma_{j}}$ is positive definite 
for all Galois embeddings $\sigma_{j}: K \hookrightarrow \mathbb{R}, j=2, \ldots, d$ which are different from the original embedding (denoted by $\sigma_{1}$ ). Thus the group $\mathrm{PO}_{f}\left(\mathcal{O}_{K}\right)$ is an arithmetic lattice in $\mathrm{PO}_{f}(\mathbb{R})$.

Let $H_{0}, \ldots, H_{k}$ be pairwise disjoint hyperplanes in $\mathcal{H}^{n}$ defined by $H_{i}=\mathcal{H}^{n} \cap\left\langle e_{i}\right\rangle^{\perp_{f}}$, $e_{i} \in K^{n+1}, i=0, \ldots, k$.

Lemma 3.1 There exists a finite index subgroup $\Gamma^{\prime}<\mathrm{PO}_{f}\left(\mathcal{O}_{K}\right)$ such that for every $h \in \Gamma^{\prime}$,

$$
\text { either } \quad h\left(H_{0}\right)=H_{0} \quad \text { or } \quad h\left(H_{0}\right) \cap\left(H_{0} \cup \cdots \cup H_{k}\right)=\varnothing \text {. }
$$

Proof Write $\Gamma=\mathrm{PO}_{f}\left(\mathcal{O}_{K}\right)$. Since multiplying the $e_{i}$ by the denominators of their entries preserves their orthogonality with the $H_{i}$, we can assume the $e_{i}$ have entries in $\mathcal{O}_{K}$. Thus, if $h \in \Gamma$, then $\left(h\left(e_{0}\right), e_{i}\right) \in \mathcal{O}_{K}$. (Throughout this proof, inner products and orthogonal complements are understood to be with respect to $f$ as in (2-1).)

Assume that $k=1$.

Let $\mathcal{P}$ be the principal ideal $\mathcal{P}=(\beta) \subset \mathcal{O}_{K}$, where $\beta=2 C\left(e_{0}, e_{1}\right)$ and $C>1$ is an integer to be determined later. The congruence subgroup $\Gamma(\mathcal{P})<\Gamma$ contains (by definition) precisely those elements $h \in \Gamma$ with the property that

$$
h \equiv \mathbf{i d}(\bmod \mathcal{P})
$$

(Here we use the fact that $\mathrm{PO}_{f}$ can be identified with the matrix group $\mathrm{O}_{f}^{\prime}$, the subgroup of the orthogonal group $\mathrm{O}_{f}$ which preserves the upper half-space.) Hence, given $h \in \Gamma(\mathcal{P})$, we find that

$$
\left(h\left(e_{0}\right), e_{1}\right)=\left(e_{0}, e_{1}\right)+\alpha \beta
$$

for some $\alpha \in \mathcal{O}_{K}$ (where $\alpha$ depends on $h$ ). We wish to show that for every $h \in \Gamma_{1}=$ $\Gamma(\mathcal{P})$ we have $h\left(H_{0}\right) \cap H_{1}=\varnothing$.

To be able to examine the intersections of the hyperplanes we use the following inequality (see Ratcliffe [15, Theorem 3.2.6]): two hyperplanes in $\mathcal{H}^{n}$, defined as above by normal vectors $v_{0}$ and $v_{1}$, intersect transversally if and only if

$$
\left|\left(v_{0}, v_{1}\right)\right|<\sqrt{\left(v_{0}, v_{0}\right)\left(v_{1}, v_{1}\right)} .
$$

So we find that the hyperplanes are disjoint or coincide completely if

$$
\left|\left(v_{0}, v_{1}\right)\right| \geqslant \sqrt{\left(v_{0}, v_{0}\right)\left(v_{1}, v_{1}\right)} .
$$


If $\alpha=0$ in (3-1) then

$$
\left|\left(h\left(e_{0}\right), e_{1}\right)\right|=\left|\left(e_{0}, e_{1}\right)\right| \geqslant \sqrt{\left(e_{0}, e_{0}\right)\left(e_{1}, e_{1}\right)}=\sqrt{\left(h\left(e_{0}\right), h\left(e_{0}\right)\right)\left(e_{1}, e_{1}\right)}
$$

(where the inequality follows from (3-2) and the initial condition $H_{0} \cap H_{1}=\varnothing$ ), and hence the hyperplanes $h\left(H_{0}\right)$ and $H_{1}$ are either disjoint or equal. We will eliminate the possibility of equality later in the proof.

If $|\alpha| \geqslant 1$, then

$$
\begin{aligned}
\left|\left(h\left(e_{0}\right), e_{1}\right)\right| & =\left|\left(e_{0}, e_{1}\right)+\alpha \beta\right| \\
& \geqslant|| \alpha|| \beta|-|\left(e_{0}, e_{1}\right)|| \\
& =\left|\left(e_{0}, e_{1}\right)\right| \cdot|2 C| \alpha|-1| \\
& >\left|\left(e_{0}, e_{1}\right)\right|
\end{aligned}
$$

(by the triangle inequality for $|\cdot|$ )

(since $C>1$ ).

The case $|\alpha|<1$ requires more care. Writing $x^{\sigma}$ for the conjugate of $x \in K$ by $\sigma \in \operatorname{Gal}(K / \mathbb{Q})$, we have the norm $N(x)$ of $x$ defined as the product $\prod_{\sigma \in \operatorname{Gal}(K / \mathbb{Q})} x^{\sigma}$ (see Lang [11, Chapter I, Section 5]); and if $x \in \mathcal{O}_{K}$ then $N(x) \in \mathbb{Z}$ so that $|N(x)| \geqslant 1$ for $x \in \mathcal{O}_{K} \backslash\{0\}$ [11, Chapter I, Corollary to Proposition 5]. Since $\alpha \in \mathcal{O}_{K} \backslash\{0\}$ and $|\alpha|<1$, the preceeding means that $\left|\alpha^{\sigma_{j}}\right|>1$ for some $j \in\{2, \ldots, d\}$. For this $j$, we get

$$
\begin{aligned}
\left|\left(h\left(e_{0}\right), e_{1}\right)^{\sigma_{j}}\right| & =\left|\left(e_{0}, e_{1}\right)^{\sigma_{j}}+\alpha^{\sigma_{j}} \beta^{\sigma_{j}}\right| \\
& \geqslant\left|\alpha^{\sigma_{j}}\right|\left|\beta^{\sigma_{j}}\right|-\left|\left(e_{0}, e_{1}\right)^{\sigma_{j}}\right||=| \beta^{\sigma_{j}}||\left|\alpha^{\sigma_{j}}\right|-\frac{1}{2 C} \mid \\
& >\frac{1}{2}\left|\beta^{\sigma_{j}}\right|=C\left|\left(e_{0}, e_{1}\right)^{\sigma_{j}}\right| .
\end{aligned}
$$

We have

$$
\left|\left(h\left(e_{0}\right), e_{1}\right)^{\sigma_{j}}\right|>C\left|\left(e_{0}, e_{1}\right)^{\sigma_{j}}\right|=C\left|\left(e_{0}, e_{1}\right)^{\sigma_{j}}\right| \frac{\left|\left(h\left(e_{0}\right), e_{1}\right)^{\sigma_{j}}\right|}{\left|\left(h\left(e_{0}\right), e_{1}\right)^{\sigma_{j}}\right|} .
$$

Now since $(\cdot,)_{f} \sigma_{j}$ is positive definite (for $j \geqslant 2$ ), the Cauchy-Schwarz inequality applies and we can use it to bound the denominator from above. (Note that we have $(u, v)^{\sigma}=\left(u^{\sigma}, v^{\sigma}\right)_{f}$.) Thus (3-4) becomes

$$
\left|\left(h\left(e_{0}\right), e_{1}\right)^{\sigma_{j}}\right|>C \frac{\left|\left(e_{0}, e_{1}\right)^{\sigma_{j}}\right|\left|\left(h\left(e_{0}\right), e_{1}\right)^{\sigma_{j}}\right|}{\sqrt{\left(h\left(e_{0}\right), h\left(e_{0}\right)\right)^{\sigma_{j}}\left(e_{1}, e_{1}\right)^{\sigma_{j}}}}=C \frac{\left|\left(e_{0}, e_{1}\right)^{\sigma_{j}}\right|\left|\left(h\left(e_{0}\right), e_{1}\right)^{\sigma_{j}}\right|}{\sqrt{\left(e_{0}, e_{0}\right)^{\sigma_{j}}\left(e_{1}, e_{1}\right)^{\sigma_{j}}}} .
$$


Multiplying each side of this inequality by all the $\left|\left(h\left(e_{0}\right), e_{1}\right)^{\sigma_{k}}\right|$ for which $k \neq j$ gives

$$
\left|N\left(\left(h\left(e_{0}\right), e_{1}\right)\right)\right|>C \underbrace{\left|N\left(\left(h\left(e_{0}\right), e_{1}\right)\right)\right|} \frac{\left|\left(e_{0}, e_{1}\right)^{\sigma_{j}}\right|}{\sqrt{\left(e_{0}, e_{0}\right)^{\sigma_{j}}\left(e_{1}, e_{1}\right)^{\sigma_{j}}}} .
$$

(*)

We can replace $(*)$ by $\left|N\left(\left(e_{0}, e_{1}\right)\right)\right|$, for

$$
\begin{aligned}
& \left|N\left(\left(h\left(e_{0}\right), e_{1}\right)\right)\right|=\left|N\left(\left(e_{0}, e_{1}\right)+2 C \alpha\left(e_{0}, e_{1}\right)\right)\right| \\
& =\left|N\left(\left(e_{0}, e_{1}\right)\right)\right| \cdot|N(1+2 C \alpha)| \quad \text { (by multiplicativity of the norm) } \\
& \geqslant\left|N\left(\left(e_{0}, e_{1}\right)\right)\right| \\
& \text { (since } 1+2 C \alpha \in \mathcal{O}_{K} \backslash\{0\} \text { ). }
\end{aligned}
$$

Writing both norms in (3-5) as products of Galois conjugates, (3-5) and (3-6) give

$$
\prod_{i=1}^{d}\left|\left(h\left(e_{0}\right), e_{1}\right)^{\sigma_{i}}\right|>C\left(\prod_{i=1}^{d}\left|\left(e_{0}, e_{1}\right)^{\sigma_{i}}\right|\right) \frac{\left|\left(e_{0}, e_{1}\right)^{\sigma_{j}}\right|}{\sqrt{\left(e_{0}, e_{0}\right)^{\sigma_{j}}\left(e_{1}, e_{1}\right)^{\sigma_{j}}}},
$$

so that by rearranging,

$$
\begin{aligned}
\left|\left(h\left(e_{0}\right), e_{1}\right)\right| & >C \frac{\left|\left(e_{0}, e_{1}\right)^{\sigma_{j}}\right|}{\sqrt{\left(e_{0}, e_{0}\right)^{\sigma_{j}}\left(e_{1}, e_{1}\right)^{\sigma_{j}}}}\left(\prod_{i=2}^{d} \frac{\left|\left(e_{0}, e_{1}\right)^{\sigma_{i}}\right|}{\left|\left(h\left(e_{0}\right), e_{1}\right)^{\sigma_{i}}\right|}\right)\left|\left(e_{0}, e_{1}\right)\right| \\
& \geqslant C \frac{\left|\left(e_{0}, e_{1}\right)^{\sigma_{j}}\right|}{\sqrt{\left(e_{0}, e_{0}\right)^{\sigma_{j}}\left(e_{1}, e_{1}\right)^{\sigma_{j}}}}\left(\prod_{i=2}^{d} \frac{\left|\left(e_{0}, e_{1}\right)^{\sigma_{i}}\right|}{\sqrt{\left(e_{0}, e_{0}\right)^{\sigma_{i}}\left(e_{1}, e_{1}\right)^{\sigma_{i}}}}\right)\left|\left(e_{0}, e_{1}\right)\right| .
\end{aligned}
$$

At this point by choosing $C$ to be sufficiently large we can ensure that $\left|\left(h\left(e_{0}, e_{1}\right)\right)\right|>$ $\left|\left(e_{0}, e_{1}\right)\right|$. Notice that since $\alpha$ depends on $h$, so too does $j$; however, we can choose $C$ independently of $h$ by assuming

$$
C \geqslant \prod_{\sigma_{j} \in S} \frac{\left(e_{0}, e_{0}\right)^{\sigma_{j}}\left(e_{1}, e_{1}\right)^{\sigma_{j}}}{\left[\left(e_{0}, e_{1}\right)^{\sigma_{j}}\right]^{2}}
$$

where $S \subseteq\left\{\sigma_{2}, \ldots, \sigma_{d}\right\}$ is the set of all $\sigma_{j}$ for which the corresponding factors in (3-8) are greater than 1 .

Thus we get $\left|\left(h\left(e_{0}\right), e_{1}\right)\right| \geqslant\left|\left(e_{0}, e_{1}\right)\right| \geqslant \sqrt{\left(h\left(e_{0}\right), h\left(e_{0}\right)\right)\left(e_{1}, e_{1}\right)}$ as in the other two cases for $\alpha$. This means that $h\left(H_{0}\right)$ either coincides with, or does not intersect $H_{1}$.

To avoid the possibility of $h\left(H_{0}\right)$ coinciding with $H_{1}$, we have to ensure that $h\left(e_{0}\right) \neq$ $\pm \omega e_{1}$ for some $\omega \in \mathbb{R}_{>0}$. If it exists, then this $\omega$ would be equal to $\sqrt{\left(e_{0}, e_{0}\right) /\left(e_{1}, e_{1}\right)}$ and there are two possible cases: 
(a) $\omega \notin K$, whence $h\left(e_{0}\right)= \pm \omega e_{1}$ is impossible.

(b) $\omega \in K$.

Let $e_{1}^{\prime}$ be the vector obtained by scaling $\omega e_{1}$ by $\sqrt{\left(e_{0}, e_{0}\right)\left(e_{1}, e_{1}\right)}$, so that $e_{1}^{\prime}=$ $\left(e_{0}, e_{0}\right) e_{1}$. Similarly, define $e_{0}^{\prime}=\sqrt{\left(e_{0}, e_{0}\right)\left(e_{1}, e_{1}\right)} e_{0}$. Thus we have $h\left(e_{0}\right)= \pm \omega e_{1}$ if and only if $h\left(e_{0}^{\prime}\right)= \pm e_{1}^{\prime}$. We verify that $e_{0}^{\prime}$ and $e_{1}^{\prime}$ are in fact in $\mathcal{O}_{K}^{n+1}$, using the following:

(1) If $x \in \mathcal{O}_{K}$ and $\sqrt{x} \in K$ then $\sqrt{x} \in \mathcal{O}_{K}$. Indeed, if $x^{m}+a_{m-1} x^{m-1}+\cdots+a_{0}=$ 0 is an equation (with coefficients in $\mathbb{Z}$ ) giving rise to the algebraic integer $x$ (see Lang [11, Chapter I, Section 2]), then $(\sqrt{x})^{2 m}+a_{m-1}(\sqrt{x})^{2(m-1)}+\cdots+a_{0}=0$ holds and hence $\sqrt{x}$ is an algebraic integer. Denote by $\mathbf{A}$ the set of all algebraic integers, and note that $\mathcal{O}_{K}=\mathbf{A} \cap K$ (see [11, Chapter 1, Proposition 5]). Thus since $\sqrt{x} \in K$ we have $\sqrt{x} \in \mathcal{O}_{K}$.

(2) If $\sqrt{x / y} \in K$ (for $x, y \in K$ ), then $\sqrt{x y} \in K$, for $\sqrt{x y}=y \sqrt{x / y} \in K$.

Now for $h\left(e_{0}^{\prime}\right)= \pm e_{1}^{\prime}$ to hold we must have $e_{0}^{\prime}+v= \pm e_{1}^{\prime}$ where $v \equiv 0$ modulo $\mathcal{P}$. If $e_{0}^{\prime}+e_{1}^{\prime}$ and $e_{0}^{\prime}-e_{1}^{\prime}$ are not congruent to 0 modulo $\mathcal{P}$, then this coincidence will not occur, and we can ensure this by choosing $C$ sufficiently large.

It remains to check that $h\left(H_{0}\right)$ and $H_{0}$ either coincide or are disjoint. One can repeat all of the above argument as far as (3-7), with $e_{0}$ in place of $e_{1}$, and we find that the ideal $\mathcal{P}^{\prime}=2\left(e_{0}, e_{0}\right)$ actually suffices in place of $\mathcal{P}$ to ensure that we have $\left|\left(h\left(e_{0}\right), e_{0}\right)\right| \geqslant \sqrt{\left(h\left(e_{0}\right), h\left(e_{0}\right)\right)\left(e_{0}, e_{0}\right)}$ for every $h \in \Gamma\left(\mathcal{P}^{\prime}\right)$. Denote $\Gamma\left(\mathcal{P}^{\prime}\right)$ by $\Gamma_{0}$.

If $k \geqslant 2$, then to separate all hyperplanes we apply the above argument to all other $e_{i}$, $(i=2, \ldots, k)$ so that we get $\Gamma_{2}, \ldots, \Gamma_{k}$ which are also finite-index subgroups of $\Gamma$. The group $\Gamma^{\prime}=\Gamma_{0} \cap \Gamma_{1} \cap \cdots \cap \Gamma_{k}$ will then satisfy the conclusion of the lemma, and is still of finite index in $\Gamma$.

Remark 3.2 If we assume that the hyperplanes $H_{0}, \ldots, H_{k}$ are not only disjoint but also do not meet at infinity then the inequality in (3-3) becomes strict and the coincidence of $h\left(H_{0}\right)$ and $H_{i}$ (for $i=1, \ldots, k$ ) is automatically avoided.

\section{Proof of Theorem 1.2}

Let $M^{\prime}$ be a compact hyperbolic $n$-manifold as in Section 2, for which $M$ is a double. By the construction, $M^{\prime}$ has a totally geodesic arc of length $\ell=\frac{1}{2} \operatorname{Syst}_{1}(M)$ with endpoints in $\partial M^{\prime}$. This value $\ell$ appears in the orthospectrum of $M^{\prime}$ as defined in the 
paper by Bridgeman and Kahn [4]. In order to bound the volume of $M^{\prime}$, and hence of $M$, we can apply the result of Bridgeman and Kahn which relates the volume and orthospectrum of a compact hyperbolic $n$-manifold with non-empty totally geodesic boundary.

Assuming $n \geqslant 3$, by [4, Theorem 1] we have $\operatorname{Vol}\left(M^{\prime}\right) \geqslant F_{n}(\ell)$, and by [4, Lemma 9(3)], $\lim _{\ell \rightarrow 0} \ell^{n-2} F_{n}(\ell)=K_{n}$, where $F_{n}$ is a continuous monotonically decreasing function $\mathbb{R}_{>0} \rightarrow \mathbb{R}_{>0}$ and $K_{n}$ is an explicit positive constant given there. It follows that there exists $K_{n}^{\prime}>0$ such that if $\ell<1$, then

$$
F_{n}(\ell) \geqslant \frac{K_{n}^{\prime}}{\ell^{n-2}}
$$

Therefore,

$$
\operatorname{Vol}(M)=2 \operatorname{Vol}\left(M^{\prime}\right) \geqslant \frac{2^{n-1} K_{n}^{\prime}}{\operatorname{Syst}_{1}(M)^{n-2}},
$$

if $\operatorname{Syst}_{1}(M)<2$.

For $\operatorname{Syst}_{1}(M) \geqslant 2$, we can refer to the Kazhdan-Margulis theorem which asserts that there is a constant $A_{n}>0$ such that $\operatorname{Vol}(M) \geqslant A_{n}$ (see Kazhdan and Margulis [10]). Hence we can take $C_{n}=\min \left(2^{n-1} K_{n}^{\prime}, 2^{n-2} A_{n}\right)$ and the theorem is proven.

Remark 4.1 It was pointed out by the referee that a similar bound holds in a more general setting if the systole of $M$ is hyperbolic. Indeed, the volume of a Margulis tube about the systole of $M$ can be bounded below by $C / \ell^{n-2}$ in the case where the corresponding element of $\pi_{1}(M)$ has no rotational part. We refer the reader to Reznikov [16] for details.

It is interesting to see how close the inequality of Theorem 1.2 approximates the actual growth of volume in our construction. In Section 2 the desired manifold $M$ is obtained as a double of $M^{\prime}$, which in turn appears as a part of the quotient manifold $L=\Lambda \backslash \mathcal{H}^{n}$. Hence

$$
\operatorname{Vol}(M)=2 \operatorname{Vol}\left(M^{\prime}\right) \leqslant 2 \operatorname{Vol}(L) .
$$

The volume of $L$ depends on the index of $\Lambda$ in the initial group $\Gamma$ which can be estimated from the proof of the generalised Margulis-Vinberg lemma in Section 3. Let us note that by the argument in Section 3 we can always make the index $|\Gamma: \Lambda|$ arbitrarily large, and hence cannot bound the volume of $L$ from above. However, we are rather interested in understanding how small the volume can get when $\epsilon$ in Theorem 1.1 is close to zero and here we can say more. 
Proposition 4.2 For every $n \geqslant 2$ there exists a sequence of manifolds $\left\{M_{i}\right\}$ from Theorem 1.1, such that when $i \rightarrow \infty, \operatorname{Syst}_{1}\left(M_{i}\right) \rightarrow 0$ and

$$
\operatorname{Vol}\left(M_{i}\right) \leqslant \frac{B_{n}}{\operatorname{Syst}_{1}\left(M_{i}\right)^{\gamma_{n}}},
$$

where $B_{n}$ and $\gamma_{n}$ are positive constants depending on $n$.

Proof Consider a sequence of inbred manifolds $M_{i}$ with $\operatorname{Syst}_{1}\left(M_{i}\right)=\epsilon_{i} \rightarrow 0$ when $i \rightarrow \infty$. For each $M_{i}$ we have associated vectors $e_{0}^{(i)}$ and $e_{1}^{(i)}$ defined in Section 2 and an ideal $\mathcal{P}^{(i)} \in \mathcal{O}_{K}$ defined in Section 3. We have

$$
\left|N\left(\mathcal{P}^{(i)}\right)\right|=\left(2 C_{i}\right)^{d}\left|N\left(\left(e_{0}^{(i)}, e_{1}^{(i)}\right)\right)\right|,
$$

where $C_{i}$ satisfies inequality (3-8). Recall that in Section 3 the vectors $e_{0}^{(i)}$ and $e_{1}^{(i)}$ are normalised so that they have coordinates in $\mathcal{O}_{K}$. This implies that when the angle between $e_{0}^{(i)}$ and $e_{1}^{(i)}$ tends to 0 , either $\left|N\left(\left(e_{0}^{(i)}, e_{1}^{(i)}\right)\right)\right|$ or the lower bound for $C_{i}$ will grow, and hence the absolute value of the norm $\left|N\left(\mathcal{P}^{(i)}\right)\right| \rightarrow \infty$.

We give a more concrete example to provide the $\left\{M_{i}\right\}$ for the conclusion of the proposition. Let $K=\mathbb{Q}(\sqrt{5})$ and $f=-\sqrt{5} x_{0}^{2}+x_{1}^{2}+\cdots+x_{n}^{2}$. The sequence of matrices

$$
A_{i}=\left(\begin{array}{ccccc}
\frac{i^{2}+\sqrt{5}}{i^{2}-\sqrt{5}} & 0 & \cdots & 0 & \frac{-2 i}{i^{2}-\sqrt{5}} \\
0 & 1 & & & 0 \\
\vdots & & \ddots & & \vdots \\
0 & & & 1 & 0 \\
\frac{-2 i \sqrt{5}}{i^{2}-\sqrt{5}} & 0 & \cdots & 0 & \frac{i^{2}+\sqrt{5}}{i^{2}-\sqrt{5}}
\end{array}\right)
$$

can be shown to lie in $\mathrm{O}_{f}^{\prime}(K)$ (the $K$-points of the subgroup of $\mathrm{O}_{f}$ that preserves $\left.\mathcal{H}^{n}\right)$, and clearly $A_{i} \rightarrow$ id as $i \rightarrow \infty$. Let $e_{0}=(0,0, \ldots, 0,1)$, so that

$$
A_{i}\left(e_{0}\right)=\left(\frac{-2 i}{i^{2}-\sqrt{5}}, 0, \ldots, 0, \frac{i^{2}+\sqrt{5}}{i^{2}-\sqrt{5}}\right) \in K^{n+1} \text {. }
$$

Rescaling $e_{0}$ and $A_{i}\left(e_{0}\right)$, we define

$$
e_{0}^{(i)}=\left(0,0, \ldots, i^{2}-\sqrt{5}\right) \quad \text { and } \quad e_{1}^{(i)}=\left(-2 i, 0, \ldots, 0, i^{2}+\sqrt{5}\right),
$$

which give

$$
\left(e_{0}^{(i)}, e_{1}^{(i)}\right)=i^{4}-5 \quad \text { and } \quad\left(e_{0}^{(i)}, e_{0}^{(i)}\right)=\left(e_{1}^{(i)}, e_{1}^{(i)}\right)=\left(i^{2}-\sqrt{5}\right)^{2} .
$$

Then $e_{0}^{(i)}$ and $e_{1}^{(i)}$ can be seen to define disjoint hyperplanes in $\mathcal{H}^{n}$ by (3-2): note that the inequality is strict. 
For our choice of $K$ there is only one nontrivial Galois automorphism $\sigma: a+b \sqrt{5} \mapsto$ $a-b \sqrt{5}$, so we also compute

$$
\left(e_{0}^{(i)}, e_{1}^{(i)}\right)^{\sigma}=i^{4}-5 \quad \text { and } \quad\left(e_{0}^{(i)}, e_{0}^{(i)}\right)^{\sigma}=\left(e_{1}^{(i)}, e_{1}^{(i)}\right)^{\sigma}=\left(i^{2}+\sqrt{5}\right)^{2} .
$$

The proof of Lemma 3.1 gives two ideals

$$
\mathcal{P}_{0}^{(i)}=\left(2\left(e_{0}^{(i)}, e_{0}^{(i)}\right)\right) \quad \text { and } \quad \mathcal{P}_{1}^{(i)}=\left(2 C\left(e_{1}^{(i)}, e_{1}^{(i)}\right)\right),
$$

and we require that

$$
\left.C \geqslant \frac{\left(i^{2}+\sqrt{5}\right)}{\left(i^{2}-\sqrt{5}\right)} \quad \text { (see }(3-8)\right) .
$$

We also need $e_{0}^{(i)} \pm e_{1}^{(i)}$ to be nonzero modulo $\mathcal{P}_{1}^{(i)}$. That is,

$$
\left(-2 i, 0, \ldots, 0,2 i^{2}\right) \text { and }(2 i, 0, \ldots, 0,-2 \sqrt{5})
$$

are not zero modulo $\mathcal{P}_{1}^{(i)}$. Since $\mathcal{P}_{1}^{(i)}=\left(2 C\left(i^{2}-\sqrt{5}\right)^{2}\right)$, this holds automatically. Observe that if $i$ is large, then $C=2$ is sufficient. Note also that since $\mathcal{P}_{0}^{(i)}$ divides $\mathcal{P}_{1}^{(i)}$, we need only consider $\mathcal{P}_{1}^{(i)}$ and can take $\Gamma_{i}^{\prime}=\Gamma\left(\mathcal{P}_{1}^{(i)}\right)$. (Actually by Remark 3.2 we needn't make this justification but it is included here for completeness of exposition.)

Note that the proof of Theorem 1.1 requires Lemma 3.1 to be applied a second time, with $e_{0}^{(i)}$ and $e_{1}^{(i)}$ interchanged. However, since both vectors are of the same length, the ideal $\mathcal{Q}_{1}^{(i)}=\left(4\left(e_{0}^{(i)}, e_{0}^{(i)}\right)\right)$ is equal to $\mathcal{P}_{1}^{(i)}$ anyway, and so we can effectively ignore this step.

Now from hyperbolic geometry, we have (see Ratcliffe [15, Theorem 3.2.8])

$$
\cosh \rho\left(H_{0}^{(i)}, H_{1}^{(i)}\right)=\frac{\left|\left(e_{0}^{(i)}, e_{1}^{(i)}\right)\right|}{\left\|e_{0}^{(i)}\right\|\left\|e_{1}^{(i)}\right\|}=\frac{i^{2}+\sqrt{5}}{i^{2}-\sqrt{5}}
$$

where $\rho$ is the distance between the hyperplanes $H_{0}^{(i)}$ and $H_{1}^{(i)}$ defined by $e_{0}^{(i)}$ and $e_{1}^{(i)}$ respectively. We see that $\rho \rightarrow 0$ as $i \rightarrow \infty$.

In the manifold $M_{i}$ obtained by the inbreeding construction, we have $\epsilon_{i}=\operatorname{Syst}_{1}\left(M_{i}\right)=$ $2 \rho_{i}$ where $\rho_{i}=\rho\left(H_{0}^{(i)}, H_{1}^{(i)}\right)$. Now, by (4-1),

$$
\epsilon_{i}=2 \cosh ^{-1}\left(\frac{i^{2}+\sqrt{5}}{i^{2}-\sqrt{5}}\right) .
$$

Using a Taylor expansion we deduce that for some constant $\delta>0$ we have

$$
\epsilon_{i} \sim \frac{\delta}{i} \quad \text { for large } i
$$


Writing $\mathcal{P}_{1}^{(i)}=(\beta)$ with $\beta=4\left(i^{2}-\sqrt{5}\right)^{2}$, we have

$$
\left|N\left(\mathcal{P}_{1}^{(i)}\right)\right|=16\left(i^{2}-\sqrt{5}\right)^{2}\left(i^{2}+\sqrt{5}\right)^{2} \sim B\left(\delta / \epsilon_{i}\right)^{8}
$$

for some constant $B>0$.

Now for a given $\mathcal{P}^{(i)}$, note that $\left|N\left(\mathcal{P}^{(i)}\right)\right|$ is the number of elements in the residue class ring $\mathcal{O}_{K} / \mathcal{P}^{(i)}$ (see Lang [11, Chapter I, Section 7]), so $\left|\Gamma: \Gamma_{i}^{\prime}\right| \leqslant\left|N\left(\mathcal{P}^{(i)}\right)\right|^{(n+1)^{2}}$ since $\left|\Gamma: \Gamma_{i}^{\prime}\right|$ is the order of a matrix group over $\mathcal{O}_{K} / \mathcal{P}^{(i)}$. Thus for some positive constant $D$,

$$
\operatorname{Vol}\left(\mathcal{H}^{n} / \Gamma_{i}^{\prime}\right)=\operatorname{Vol}\left(\mathcal{H}^{n} / \Gamma\right) \cdot\left|\Gamma: \Gamma_{i}^{\prime}\right| \leqslant D\left(B\left(\delta / \epsilon_{i}\right)^{8}\right)^{(n+1)^{2}},
$$

which is a polynomial in $1 / \operatorname{Syst}_{1}\left(M_{i}\right)$ of degree $8(n+1)^{2}$.

Other related constructions of extremal sequences of manifolds $\left\{M_{i}\right\}$ are given in detail in the second author's forthcoming PhD thesis [17].

\section{Remarks}

\subsection{Arithmeticity}

If $\epsilon$ in Theorem 1.1 is less than some $\epsilon_{0}>0$, which depends only on the degree $d$ of the field $K$ in the construction, and on the dimension $n$, then the manifolds $M$ produced by the theorem are non-arithmetic. This can be seen as follows: Assume that the manifold $M$ is arithmetic. The fundamental group $\pi_{1}\left(M^{\prime}\right)$ (of the compact manifold $M^{\prime}$ with boundary, of which $M$ is a double) injects into $\pi_{1}(M)$ and both are Zariski dense in $\operatorname{PO}(n, 1)^{\circ}$. This is shown by Gromov and Piatetski-Shapiro [7, Sections 0.2 and 1.7]. By the commensurability criterion [7, Section 1.6] we conclude that $\Gamma_{M}=\pi_{1}(M)$ is commensurable with $\mathrm{PO}_{f}\left(\mathcal{O}_{K}\right)$. Now we can follow a known argument relating the lengths of geodesics of an arithmetic manifold $M$ to eigenvalues of integral matrices (see Gelander [6, Section 10]). This implies in our case that $\operatorname{Syst}_{1}(M) \geqslant C_{n, d}$, since the integral polynomials which arise have their degree bounded above by $d(n+1)$. Hence if $\epsilon<C_{n, d}$, then $M$ has to be non-arithmetic.

A conjecture of Lehmer from number theory claims that there exists a constant $m>1$ such that the Mahler measure $M(P)$ of any non-cyclotomic integral monic polynomial $P$ satisfies $M(P) \geqslant m$. (Recall that the Mahler measure of an integral monic polynomial $P$ of degree $d$ with roots $\theta_{1}, \theta_{2}, \ldots, \theta_{d}$ is defined by $M(P)=\prod_{i=1}^{d} \max \left(1,\left|\theta_{i}\right|\right)$.) Our argument shows that if Lehmer's conjecture is true then $\epsilon_{0}$ in the statement above is an absolute constant which does not depend on 
$K$ or $n$. We refer to Margulis [13, page 322] and Gelander [6, Section 10] for a related discussion addressing arithmetic manifolds.

We have shown that our method provides a new construction of non-arithmetic hyperbolic $n$-manifolds for every dimension $n$. It has some similarities with the interbreeding construction of Gromov and Piatetski-Shapiro [7] but at the same time is different from the former. Following Agol [1] we call it an inbreeding construction.

\subsection{Commensurability}

If $\epsilon \rightarrow 0$ then at most finitely many of the manifolds $M$ provided by Theorem 1.1 will be commensurable to each other. Indeed, assume that we have an infinite sequence of commensurable non-arithmetic manifolds $M_{1}=\Lambda_{1} \backslash \mathcal{H}^{n}, M_{2}=\Lambda_{2} \backslash \mathcal{H}^{n}, \ldots$, such that $\operatorname{Syst}_{1}\left(M_{i}\right)=\epsilon_{i} \rightarrow 0$ when $i \rightarrow \infty$. By Margulis' Theorem [13, Theorem 1, page 2], the commensurability group $\Gamma$ of $\Lambda_{i}$ will be a lattice in $\operatorname{Isom}\left(\mathcal{H}^{n}\right)$ and hence $\Gamma \backslash \mathcal{H}^{n}$ is a compact hyperbolic $n$-orbifold. We have

$$
\Gamma \supset \Lambda_{1}, \Lambda_{2}, \ldots,
$$

so the orbifold $\Gamma \backslash \mathcal{H}^{n}$ has systoles of arbitrarily short length, which is impossible. Note that this argument works for any non-arithmetic manifolds with short geodesics, not only those provided by our theorem.

\subsection{Non-compact manifolds}

The notion of systole being defined as the length of a shortest closed geodesic in a manifold $M$ generalises to non-compact finite volume hyperbolic $n$-manifolds. In terms of the lattice $\Lambda \subset \operatorname{Isom}\left(\mathcal{H}^{n}\right)$ uniformising $M$, the closed geodesics in $M$ correspond to the hyperbolic elements of $\Lambda$, while $\Lambda$ also contains parabolics which have zero displacement and correspond to the cusps. With these observations at hand the results of this paper can be generalised to the finite volume non-compact hyperbolic $n$-manifolds. The proofs are entirely similar and we omit them.

\subsection{Some applications}

Our non-arithmetic manifolds $M$ contain properly embedded separating totally geodesic hypersurfaces, and hence the fundamental group $\pi_{1}(M)$ has the structure of a free product with amalgamated subgroup similar to the fundamental groups of the Gromov-Piatetski-Shapiro manifolds [7]. This enables one to use our manifolds for the construction of Belolipetsky and Lubotzky [3], which proves that every finite group can be realised as the full isometry group of some compact hyperbolic $n$-manifold. 
Another immediate application is to the construction of some new non-coherent lattices in $\operatorname{Isom}\left(\mathcal{H}^{n}\right)$, which can be achieved by applying the argument of Kapovich-PotyagailoVinberg [8, Section 4] to the lattices provided by our construction. The details of this application are explained in the second author's forthcoming $\mathrm{PhD}$ thesis [17].

\subsection{Other locally symmetric spaces and a conjecture of Lehmer}

It is natural to ask what can be said about systoles of other locally symmetric manifolds. This question pertains to the quotients of symmetric spaces $X=H / K$ by torsion-free lattices, where $H$ is now a semisimple Lie group and $K$ its maximal compact subgroup.

If all lattices in $H$ are arithmetic and Lehmer's conjecture (or its weaker version by Margulis [13, Chapter IX, Section 4.21]) holds, then the systoles of the $X$-locally symmetric manifolds will be bounded below by a constant which depends only on $X$ (see Gelander [6, Section 10]). The arithmeticity of lattices is known for groups of real rank at least 2 by Margulis [13, Theorem 1, page 2], and for $H=\operatorname{Sp}(n, 1)$ or $\mathrm{F}_{4}^{-20}$ by Corlette [5]. Hence the only case for which one may hope to have a version of our result is when $H=\mathrm{PU}(n, 1)$ and $X$ is complex hyperbolic $n$-space.

There is also a reverse connection between Lehmer's conjecture and systoles of arithmetic locally symmetric manifolds. This is explained in detail for the low dimensional hyperbolic manifolds in Machlachlan and Reid [12, Chapter 12.3] and the report by the first author [2].

\section{References}

[1] I Agol, Systoles of hyperbolic 4-manifolds arXiv:math/0612290

[2] M Belolipetsky, Geodesics, volumes and Lehmer's conjecture, from: "LowDimensional Topology and Number Theory”, Oberwolfach Reports 7 (2010) 2136-2139

[3] M Belolipetsky, A Lubotzky, Finite groups and hyperbolic manifolds, Invent. Math. 162 (2005) 459-472 MR2198218

[4] M Bridgeman, J Kahn, Hyperbolic volume of manifolds with geodesic boundary and orthospectra, Geom. Funct. Anal. 20 (2010) 1210-1230 MR2746952

[5] K Corlette, Archimedean superrigidity and hyperbolic geometry, Ann. of Math. (2) 135 (1992) 165-182 MR1147961

[6] T Gelander, Homotopy type and volume of locally symmetric manifolds, Duke Math. J. 124 (2004) 459-515 MR2084613

[7] M Gromov, I Piatetski-Shapiro, Nonarithmetic groups in Lobachevsky spaces, Inst. Hautes Études Sci. Publ. Math. (1988) 93-103 MR932135 
[8] M Kapovich, L Potyagailo, E Vinberg, Noncoherence of some lattices in $\operatorname{Isom}\left(\mathbb{H}^{n}\right)$, from: "The Zieschang Gedenkschrift", Geom. Topol. Monogr. 14, Geom. Topol. Publ., Coventry (2008) 335-351 MR2484708

[9] M G Katz, Systolic geometry and topology, Mathematical Surveys and Monographs 137, American Mathematical Society, Providence, RI (2007) MR2292367 With an appendix by Jake P Solomon

[10] D A Kazhdan, G A Margulis, A proof of Selberg's hypothesis, Mat. Sbornik 75 (117) (1968) 162-168

[11] S Lang, Algebraic number theory, Addison-Wesley Publishing Co., Reading, MALondon-Don Mills, Ont. (1970) MR0282947

[12] C Maclachlan, A W Reid, The arithmetic of hyperbolic 3-manifolds, Graduate Texts in Mathematics 219, Springer, New York (2003) MR1937957

[13] G A Margulis, Discrete subgroups of semisimple Lie groups, Ergebnisse der Mathematik und ihrer Grenzgebiete (3) 17, Springer, Berlin (1991) MR1090825

[14] G A Margulis, È B Vinberg, Some linear groups virtually having a free quotient, J. Lie Theory 10 (2000) 171-180 MR1748082

[15] J G Ratcliffe, Foundations of hyperbolic manifolds, second edition, Graduate Texts in Mathematics 149, Springer, New York (2006) MR2249478

[16] A Reznikov, The volume and the injectivity radius of a hyperbolic manifold, Topology 34 (1995) 477-479 MR1318888

[17] S A Thomson, PhD thesis, Durham University (in preparation)

[18] W P Thurston, The geometry and topology of three-manifolds, lecture notes, Princeton University (1980) Available at http://library.msri.org/books/gt3m/

[19] H C Wang, Topics on totally discontinuous groups, from: "Symmetric spaces (Short Courses, Washington Univ., St. Louis, Mo., 1969-1970)", Pure and Appl. Math. 8, Dekker, New York (1972) 459-487 MR0414787

MVB, SAT: Department of Mathematical Sciences, Durham University South Road, Durham, DH1 3LE, United Kingdom

MVB: Institute of Mathematics, Koptyuga 4, 630090 Novosibirsk, Russia mikhail.belolipetsky@durham.ac.uk, s.a.thomson@durham.ac.uk

Received: 4 October 2010 Revised: 24 January 2011 